\title{
Induced modules for orbifold vertex operator algebras
}

\author{
By Ching Hung LAM
}

(Received Feb. 23, 1999)

(Revised Jan. 12, 2000)

\begin{abstract}
Let $V$ be a simple vertex operator algebra and $G<$ Aut $V$ a finite abelian subgroup such that $V^{G}$ is rational. We study the representations of $V$ based on certain assumptions on $V^{G}$-modules. We prove a decomposition theorem for irreducible $V$-modules. We also define an induced module from $V^{G}$ to $V$ and show that every irreducible $V$-module is a quotient module of some induced module. In addition, we prove that $V$ is rational in this case.
\end{abstract}

\section{Introduction.}

Let $V$ be a vertex operator algebra (VOA) over the field of complex numbers $C$ and $G$ a finite abelian subgroup of automorphism of $V$. The space of fixed points of $G$ in $\mathrm{V}$, denoted by $V^{G}$, is often called an orbifold vertex operator algebra. In [7], Dong and Mason proved that if $V$ is a simple VOA, then the orbifold vertex operator algebra $V^{G}$ is also a simple VOA. Moreover, as a $V^{G}$-module, $V$ decomposes as

$$
V=\bigoplus_{\chi \in \operatorname{Irr} G} V^{\chi}
$$

where $V^{\chi}$ is the sum of all irreducible $G$-modules which afford the character $\chi \in \operatorname{Irr} G$. They also showed that $V^{\chi} \neq 0$ for any $\chi \in \operatorname{Irr} G$ and $V^{\chi}$ is irreducible as a $V^{G}$-module. In addition, certain general properties related to the intertwining operators among the $V^{G}$ modules $V^{\chi}, \chi \in \operatorname{Irr} G$, were proved, which are fundamental for our work.

In this paper, we shall study the representations of $V$ based on certain assumptions on $V^{G}$ and the $V^{G}$-modules $V^{\chi}, \chi \in \operatorname{Irr} G$. Actually, we shall only consider a very simple case:

(1) $V$ is a simple VOA (i.e., $V$ is irreducible as a $V$-module) over $\boldsymbol{C}$;

(2) there exists a finite abelian subgroup $G<$ Aut $V$ such that $V^{G}$ is rational and for any irreducible $V^{G}$-module $L$, the tensor product $V^{\chi} \hat{\otimes}_{V^{G}} L$ (cf. Definition 2.10) is irreducible as a $V^{G}$-module where $V^{\chi}$ is equal to the sum of all irreducible $G$-modules affording the character $\chi$.

2000 Mathematics Subject Classification. Primary 17B69.

Key Words and Phrases. induced module, orbifold theory, rational vertex operator algebra. 
Under these assumptions, we shall obtain a decomposition of an irreducible $V$-modules into a direct sum of irreducible $V^{G}$-modules (see Theorem 3.7 and Theorem 3.11). An induced module will also be defined. In fact, we shall show that the $V^{G}$-module

$$
X_{L}:=\sum_{\chi \in \operatorname{Irr} G} V^{\chi} \hat{\otimes}_{V^{G}} L
$$

has a natural $V$-module structure where $L$ is an irreducible $V^{G}$ module and $V^{\chi} \hat{\otimes}_{V^{G}} L$ is the tensor product for $V^{\chi}$ and $L$. We call such a module $X_{L}$ an induced module and show that every irreducible $V$-module is a quotient module of some induced module. Moreover, we shall prove that $V$ is rational in this case.

One important example is the code VOA constructed by Miyamoto [12] and [13], which is also our main motivation. In fact, our main theorems (Theorem 3.11 and Theorem 4.4) are essentially generalizations for Theorem 5.1 and 5.2 of [13]. See also [2] in which a class of VOAs called framed VOAs was studied. The proof of the rationality theorem (Theorem 4.5) is basically taken from there.

This paper will be organized as follows: in Section 2, we shall review some basic definitions and notation. We shall also state our assumptions. In Section 3 , we shall study the representations of a VOA $V$ based on the structure of a finite abelian subgroup $G<$ Aut $V$ of automorphisms and its fixed point space $V^{G}$. We shall also prove a decomposition theorem for irreducible $V$ modules. In Section 4, an induced module will be defined. We shall show that every irreducible $V$-module is a quotient module of some induced module. Finally, we shall prove that $V$ is rational under certain assumptions on the fusion rules of $V^{G}$.

\section{Basic definitions.}

In this section, we shall review some basic definitions and terminology. Unless stated otherwise, we shall always work on the field of complex numbers C.

Definition 2.1 (cf. [9], [8]). A vertex operator algebra (VOA) is a $Z$-graded vector space $V=\amalg_{n \in Z} V_{n}$ equipped with a linear map

$$
\begin{aligned}
Y(, z): V & \rightarrow \text { End } V\left[\left[z, z^{-1}\right]\right] \\
v & \rightarrow Y(v, z)=\sum_{i \in \boldsymbol{Z}} v_{i} z^{-i-1}
\end{aligned}
$$

such that the following conditions hold:

(1) (Vacuum condition) there is a vector 1 such that

$$
Y(1, z)=\left.i d\right|_{V}
$$


(2) (Creation property) $Y(v, z) \cdot 1 \in V[[z]]$ and $\lim _{z \rightarrow 0} Y(v, z) \cdot 1=v$ for any $v \in V$ (i.e., $Y(v, z) \cdot 1$ involves only non-negative integral powers of $z$ and the constant term is $v$ );

(3) $\operatorname{dim} V_{n}<\infty$ and $V_{n}=0$ for sufficiently small $n$;

(4) for any $u, v \in V$,

$$
u_{n} v=0 \text { for } n \text { sufficiently large; }
$$

(5) (Virasoro condition) there is a vector $\omega$ such that the operators $L_{i}=\omega_{i+1}$ satisfy the Virasoro relation:

$$
\left[L_{m}, L_{n}\right]=(m-n) L_{m+n}+\frac{1}{12}\left(m^{3}-m\right) \delta_{m+n, 0} c
$$

where $c$ is a scalar and is called the rank of $V$;

(6) $L_{0} v=n v=(w t v) v$ for $v \in V_{n}$;

(7) $\left(L_{-1}\right.$-derivative property)

$$
Y\left(L_{-1} v, z\right)=\frac{d}{d z} Y(v, z) ;
$$

(8) (Jacobi Identity) for any $u, v \in V$,

$$
\begin{aligned}
& z_{0}^{-1} \delta\left(\frac{z_{1}-z_{2}}{z_{0}}\right) Y\left(u, z_{1}\right) Y\left(v, z_{2}\right)-z_{0}^{-1} \delta\left(\frac{-z_{2}+z_{1}}{z_{0}}\right) Y\left(v, z_{2}\right) Y\left(u, z_{1}\right) \\
& \quad=z_{2}^{-1} \delta\left(\frac{z_{1}-z_{0}}{z_{2}}\right) Y\left(Y\left(u, z_{0}\right) v, z_{2}\right) .
\end{aligned}
$$

Remark 2.2. The Jacobi identity (8) can be equivalently replaced by the following commutativity (see Dong and Lepowsky [3] and $\mathrm{Li}$ [10]):

$$
\left(z_{1}-z_{2}\right)^{n}\left(Y\left(u, z_{1}\right) Y\left(v, z_{2}\right)-Y\left(v, z_{2}\right) Y\left(u, z_{1}\right)\right)=0
$$

for a sufficiently large positive integer $n$. Here, $n$ depends on both $u$ and $v$.

We can define the notion of modules in a similar way.

Definition 2.3. A module for a VOA is a $\boldsymbol{Q}$-graded vector space $M=\amalg_{n \in Q} M_{n}$ equipped with a linear map

$$
\begin{aligned}
Y_{M}(, z): V & \rightarrow \text { End } M\left[\left[z, z^{-1}\right]\right] \\
v & \rightarrow Y_{M}(v, z)=\sum_{i \in Z} v_{i} z^{-i-1}
\end{aligned}
$$

such that all the conditions mentioned in Definition 2.1 also hold, provided that they make sense. 
REMARK 2.4. As in remark 2.2, the Jacobi identity for modules can also be equivalently replaced by the following conditions:

(1) (Commutativity) For any $u, v \in V$,

$$
\left(z_{1}-z_{2}\right)^{n}\left(Y_{M}\left(u, z_{1}\right) Y_{M}\left(v, z_{2}\right)-Y_{M}\left(v, z_{2}\right) Y_{M}\left(u, z_{1}\right)\right)=0
$$

for $n$ sufficiently large.

(2) (Associativity) For any $u, v \in V$,

$$
\begin{aligned}
& \left(z_{2}+z_{0}\right)^{k} Y_{M}\left(Y\left(u, z_{0}\right) v, z_{2}\right) w \\
& \quad=\left(z_{0}+z_{2}\right)^{k} Y_{M}\left(u, z_{0}+z_{2}\right) Y_{M}\left(v, z_{0}\right) w
\end{aligned}
$$

for some sufficiently large positive integer $k$.

We shall note that the factors $\left(z_{2}+z_{0}\right)^{k}$ and $\left(z_{0}+z_{2}\right)^{k}$ are to be expanded as a formal power series in the second variable, i.e.,

$$
\begin{aligned}
& \left(z_{2}+z_{0}\right)^{k}=\sum_{i \geq 0}\left(\begin{array}{c}
k \\
i
\end{array}\right) z_{2}^{k-i} z_{0}^{i}, \\
& \left(z_{0}+z_{2}\right)^{k}=\sum_{i \geq 0}\left(\begin{array}{c}
k \\
i
\end{array}\right) z_{0}^{k-i} z_{2}^{i} .
\end{aligned}
$$

In general, $\left(z_{2}+z_{0}\right)^{k} \neq\left(z_{0}+z_{2}\right)^{k}$; however, they agree with each other if $k$ is a positive integer.

REMARK 2.5. For computation, it is sometimes more convenient to use the following form of the Jacobi Identity (also called the Borcherds Identity): For any $a, b \in V, c \in M$ and $m, n, q \in \boldsymbol{Z}$,

$$
\sum_{i \geq 0}\left(\begin{array}{c}
m \\
i
\end{array}\right)\left(a_{q+i} b\right)_{m+n-i} c=\sum_{i \geq 0}(-1)^{i}\left(\begin{array}{c}
q \\
i
\end{array}\right)\left(a_{m+q-i} b_{n+i} c-(-1)^{q} b_{n+q-i} a_{m+i} c\right) .
$$

DEFINITION 2.6. Let $V$ be a vertex operator algebra. $V$ is said to be rational if the following conditions hold:

(1) $V$ has only finitely many inequivalent irreducible modules;

(2) every $V$-module is a direct sum of irreducible ones.

REMARK 2.7. Our definition of rationality basically follows the definition given by $\mathrm{Zhu}$ in [14]. For other definitions of rationality, please refer to Dong, $\mathrm{Li}$ and Mason's paper [4].

Definition 2.8. Let $(V, Y)$ be a VOA and let $\left(W^{1}, Y^{1}\right),\left(W^{2}, Y^{2}\right)$ and $\left.\begin{array}{l}\left(W^{3}, Y^{3}\right) \text { be } V \text {-modules. An intertwining operator of type }\left(\begin{array}{c}W^{1} \\ W^{2}\end{array} W^{3}\right.\end{array}\right)$ is a 


$$
\begin{aligned}
I(, z): W^{2} & \rightarrow \operatorname{Hom}\left(W^{3}, W^{1}\right)\{z\} \\
u & \rightarrow I(u, z)=\sum_{n \in \boldsymbol{Q}} u_{n} z^{-n-1}
\end{aligned}
$$

satisfying:

(1) for any $u \in W^{2}$ and $v \in W^{3}$,

$$
u_{n} v=0 \text { for } n \text { sufficiently large; }
$$

(2) $\quad I\left(L_{-1} v, z\right)=(d / d z) I(v, z)$;

(3) (Jacobi Identity) for any $u \in V, v \in W^{2}$

$$
\begin{aligned}
& z_{0}^{-1} \delta\left(\frac{z_{1}-z_{2}}{z_{0}}\right) Y^{1}\left(u, z_{1}\right) I\left(v, z_{2}\right)-z_{0}^{-1} \delta\left(\frac{-z_{2}+z_{1}}{z_{0}}\right) I\left(v, z_{2}\right) Y^{3}\left(u, z_{1}\right) \\
& =z_{2}^{-1} \delta\left(\frac{z_{1}-z_{0}}{z_{2}}\right) I\left(Y^{2}\left(u, z_{0}\right) v, z_{2}\right) .
\end{aligned}
$$

The set of all intertwining operators of type $\left(\begin{array}{c}W^{1} \\ W^{2}\end{array} W^{3}\right)$ is denoted by

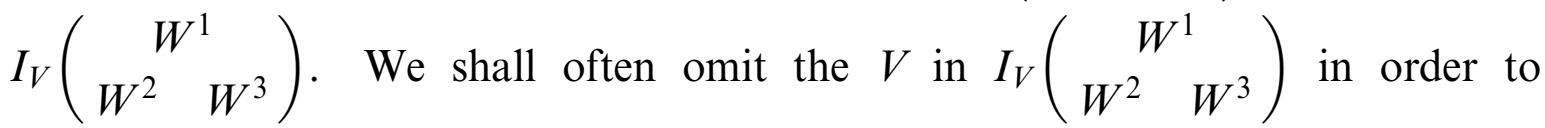
simplify the notation.

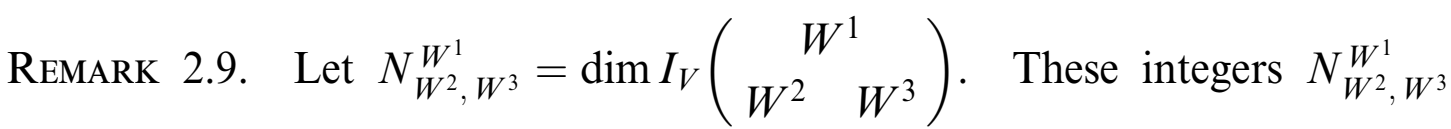
are usually called the "fusion rules". For convenience, we shall often consider the fusion product

$$
W^{2} \times W^{3}=\sum_{W} N_{W^{2}, W^{3}}^{W} W
$$

where $W$ runs over the set of equivalence classes of irreducible $V$-modules.

Definition 2.10 (cf. Li [11]). Let $M_{1}$ and $M_{2}$ be two $V$-modules. A tensor product for the ordered pair $\left(M_{1}, M_{2}\right)$ is a pair $(M, F(\cdot, z))$ consisting of a $V$ module $M$ and an intertwining operator $F(\cdot, z)$ of type $\left(\begin{array}{cc}M \\ M_{1} & M_{2}\end{array}\right)$ such that the following universal property holds: For any $V$-module $W$ and any intertwining operator $I(\cdot, z)$ of type $\left(\begin{array}{cc}W \\ M_{1} & M_{2}\end{array}\right)$, there is a unique $V$-homomorphism $\psi: M \rightarrow W$ such that $I(\cdot, z)=\psi \circ F(\cdot, z)$. We shall denote the tensor product for $\left(M_{1}, M_{2}\right)$ by $M_{1} \hat{\otimes}_{V} M_{2}$ or simply by $M_{1} \hat{\otimes} M_{2}$.

Proposition 2.11 (cf. [11]). If $V$ is rational and $M_{1}$ and $M_{2}$ are irreducible $V$-modules, then the tensor product for $\left(M_{1}, M_{2}\right)$ always exists. Moreover, 


$$
M_{1} \hat{\otimes}_{V} M_{2} \cong \bigoplus_{W} N_{M_{1}, M_{2}}^{W} W
$$

where $W$ runs over the set of equivalence classes of irreducible V-modules. In this case, the tensor product for $\left(M_{1}, M_{2}\right)$ agrees with the fusion product $M_{1} \times M_{2} . \quad B y$ abuse of notations, we may sometimes denote the tensor product by $M_{1} \times M_{2}$, also.

The following proposition is also quoted from $\mathrm{Li}$ [11].

Proposition 2.12. Let $V$ be a rational VOA and

$$
\begin{aligned}
& M^{1}=\bigoplus_{i} M_{i}^{1}, \\
& M^{2}=\bigoplus_{j} M_{j}^{2}
\end{aligned}
$$

where $M_{i}^{1}, M_{j}^{2}$ are irreducible $V$-modules. Then, the tensor product for $\left(M^{1}, M^{2}\right)$ exists and

$$
M^{1} \hat{\otimes}_{V} M^{2}=\bigoplus_{i, j} M_{i}^{1} \hat{\otimes}_{V} M_{j}^{2} .
$$

In this paper, we shall consider a simple vertex operator algebra $V$ and a finite abelian subgroup $G<$ Aut $V$. By a theorem of Dong and Mason [7], we know that the fixed point space $V^{G}$ is a simple VOA; moreover, as a $V^{G}$-module,

$$
V=\oplus_{\chi \in \operatorname{Irr} G} V^{\chi}
$$

where $V^{\chi}$ is the sum of all irreducible $G$-submodules of $V$ affording the character $\chi$ and $V^{\chi}$ is also irreducible as a $V^{G}$-module. As we assume that $G$ is abelian, the set of all irreducible character $\operatorname{Irr} G$ also forms a group by pointwise multiplication. This group will be denoted by $G^{*}$.

$V^{G}$ is always assumed to be rational in this paper. Moreover, we shall assume that the fusion rules for $V^{\chi}, \chi \in \operatorname{Irr} G$, satisfy the condition:

A1: For any fixed $\chi \in \operatorname{Irr} G$ and irreducible $V^{G}$-module $L$,

$$
\sum_{W} N_{V^{x} L}^{W}=1
$$

where $W$ runs over the set all equivalence classes of irreducible $V^{G}$-modules.

Remark 2.13. By Proposition 2.11, if $M_{1}$ and $M_{2}$ are irreducible, $M_{1} \hat{\otimes}_{V} M_{2} \cong \bigoplus_{W} N_{M_{1}, M_{2}}^{W} W$. Therefore, our assumption on the fusion rules is equivalent to the fact that $V^{\chi} \hat{\otimes}_{V^{G}} L$ is irreducible for any $\chi \in \operatorname{Irr} G$ and irreducible $V^{G}$-module $L$. Moreover, we have

$$
N_{V^{\chi} L}^{W}=\operatorname{dim} I\left(\begin{array}{c}
W \\
V^{\chi}
\end{array}\right) \leq 1
$$

for any $\chi \in \operatorname{Irr} G$ and irreducible $V^{G}$-modules $L$ and $W$. 


\section{3. $\quad V$-modules.}

In this section, we shall study the representations of $V$ based on the knowledge of $V^{G}$ and its representations. In particular, we shall prove our main theorem which describes the structure of irreducible $V$-modules.

As in the last section, $V$ denotes a simple VOA and $G<$ Aut $V$ a finite abelian subgroup. We shall assume that $V^{G}$ is rational and for any fixed $\chi \in \operatorname{Irr} G$ and irreducible $V^{G}$-module $L$,

$$
\sum_{W} N_{V \times L}^{W}=1
$$

The following proposition is very important to our discussion.

Proposition 3.1 (Proposition 11.9, [3]). Let $W^{1}, W^{2}$ and $W^{3}$ be V-modules and $I$ be an intertwining operator of type $\left(\begin{array}{cc}W^{3} \\ W^{1} & W^{2}\end{array}\right)$. Assume that $W^{1}$ and $W^{2}$ are irreducible. Then, for any $v^{1} \in W^{1}$ and $v^{2} \in W^{2}$,

$$
I\left(v^{1}, z\right) v^{2}=0 \text { implies } I(\cdot, z)=0 .
$$

Definition 3.2. Let $M$ be an irreducible $V$-module and $L$ a $V^{G}$-submodule of $M$. Define

$$
V^{\chi} \cdot L=\operatorname{span}\left\{v_{n} w \mid v \in V^{\chi} \text { and } w \in L\right\}, \quad \chi \in \operatorname{Irr} G .
$$

For convenience, we shall often denote $V^{\chi} \cdot L$ by $\chi L$ or $L^{\chi}$.

Lemma 3.3. $V^{\chi} \cdot L$ is a non-zero $V^{G}$-submodule of $M$.

Proof. Since both $V$ and $M$ are irreducible $V$-modules, it is clear that $V^{\chi} \cdot L \neq 0$ by Proposition 3.1.

Now, let $u \in V^{G}, v \in V^{\chi}$ and $w \in L$. By the Borcherds Identity (2.5) with $q=0$, we have

$$
u_{n}\left(v_{m} w\right)-v_{m}\left(u_{n} w\right)=\sum_{i \geq 0}\left(\begin{array}{c}
m \\
i
\end{array}\right)\left(u_{i} v\right)_{m+n-i} w .
$$

Since $u_{n} w \in L$ and $u_{i} v \in V^{\chi}$, we obtain $u_{n}\left(v_{m} w\right) \in V^{\chi} \cdot L$ and thus $V^{\chi} \cdot L$ is a $V^{G}$-submodule of $M$.

REMARK 3.4. Note that the module vertex operator $\left.Y_{M}(\cdot, z)\right|_{V x}$ defines a nonzero $V^{G}$-intertwining operator of type $\left(\begin{array}{cc}V^{\chi} \cdot L \\ V^{\chi} & L\end{array}\right)$. Therefore, by the 
definition of tensor product (cf. Definition 2.10), there exists a nonzero $V^{G_{-}}$ homomorphism $\phi: V^{\chi} \hat{\otimes}_{V^{G}} L \rightarrow V^{\chi} \cdot L$ such that

$$
\left.Y_{M}(\cdot, z)\right|_{V \chi}=\phi \circ F
$$

where $\left(V^{\chi} \hat{\otimes}_{V^{G}} L, F\right)$ is the tensor product for the pair $\left(V^{\chi}, L\right)$.

Since $V^{\chi} \cdot L=\operatorname{span}\left\{v_{n} w \mid v \in V^{\chi}\right.$ and $\left.w \in L\right\}, \phi$ is surjective. If $L$ is also an irreducible $V^{G}$-module, then $V^{\chi} \hat{\otimes}_{V^{G}} L$ is an irreducible $V^{G}$-module, by our assumptions. In this case, $V^{\chi} \cdot L$ must be irreducible as a $V^{G}$-module and isomorphic to $V^{\chi} \hat{\otimes}_{V^{G}} L$.

The following lemma is an easy consequence of the Borcherds identity.

Lemma 3.5. Let $M$ be an irreducible $V$-module and $L \subset M a V^{G}$-module. Then, for $\chi, \lambda \in \operatorname{Irr} G$,

(1) $V^{\chi} \cdot\left(V^{\lambda} \cdot L\right)=\left(V^{\chi} \cdot V^{\lambda}\right) \cdot L$,

(2) $V^{\chi} \cdot\left(V^{\lambda} \cdot L\right)=V^{\lambda} \cdot\left(V^{\chi} \cdot L\right)$.

Definition 3.6. Let $M$ be an irreducible $V$-module and $L$ an irreducible $V^{G}$-submodule of $M$. Define $\tilde{L}$ to be the sum of all irreducible submodules of $M$ isomorphic to $L$. $\tilde{L}$ is called a $V^{G}$-homogeneous component of $L$ in $M$.

THEOREM 3.7. Let $V$ be a simple VOA, $G<$ Aut $V$ a finite abelian subgroup such that $V^{G}$ is rational and $V^{\chi} \hat{\otimes}_{V^{G}} L$ is irreducible for any irreducible $V^{G}$ module $L$ and $\chi \in \operatorname{Irr} G$. Let $M$ be an irreducible V-module and $L$ an irreducible $V^{G}$-submodule of $M$. Then,

(1) there exists $\left\{\chi_{i}\right\} \subset \operatorname{Irr} G$ such that $M \cong \bigoplus_{i} V^{\chi_{i}} \cdot L$.

(2) All $V^{G}$-homogeneous components are permuted transitively by $G^{*}=$ $\operatorname{Irr} G$, i.e., if $\tilde{L}$ and $\tilde{L}^{\prime}$ are $V^{G}$-homogeneous components in $M$, then there is a $\chi \in \operatorname{Irr} G$ such that $\tilde{L}=V^{\chi} \cdot \tilde{L}^{\prime}$.

(3) There is a subgroup $H<G$ such that all $V^{G}$-homogeneous components in $M$ are irreducible $V^{H}$-modules. Moreover,

$$
M=\bigoplus_{\chi \in \operatorname{Irr} H} \chi \tilde{L}
$$

where $\chi \tilde{L}=V^{\chi} \cdot \tilde{L}$.

(4) Let $m(L)$ be the multiplicity of $L$ in $M$, i.e., $\tilde{L}=m(L) \cdot L$. Then, $m(L)$ $=m\left(L^{\prime}\right)$ for any irreducible $V^{G}$ submodules $L$ and $L^{\prime}$ in $M$. Moreover, we have

$$
M=\bigoplus_{i} m \cdot\left(\chi_{i} L\right)
$$

where $\chi_{i}$ is defined as in (1) and $m=m(L)$.

ProOF. (1) Let 


$$
V \cdot L=\operatorname{span}\left\{u_{n} v \mid u \in V, v \in L\right\} .
$$

Then, $M=V \cdot L$ as $M$ is an irreducible $V$-module.

Since $V=\bigoplus_{\chi \in \operatorname{Irr} G} V^{\chi}$ (cf. Dong and Mason [7]), we have

$$
M=V \cdot L=\sum_{\chi \in \operatorname{Irr} G} V^{\chi} \cdot L .
$$

By our assumption, $V^{G}$ is rational and $\left\{V^{\chi} \cdot L\right\}$ are irreducible $V^{G}$-modules. Hence, there exists a set of irreducible $G$-characters $\left\{\chi_{i}\right\}_{i} \subset \operatorname{Irr} G$ such that

$$
M=\bigoplus_{i} V^{\chi_{i}} \cdot L
$$

and we have (1).

(2) Let $L$ and $L^{\prime}$ be irreducible $V^{G}$-submodules of $M$. Then, by (1), there is a $\chi \in \operatorname{Irr} G$ such that $L^{\prime} \cong V^{\chi} \cdot L$. Thus, we also have $L^{\prime}=V^{\chi} \hat{\otimes}_{V^{G}} L$ (cf. Remark 3.4).

Let $S \subset \tilde{L}$ be an irreducible $V^{G}$-submodule. Then, $S \cong L$ and $V^{\chi} \cdot S \cong$ $V^{\chi} \hat{\otimes}_{V^{G}} S \cong V^{\chi} \hat{\otimes}_{V^{G}} L \cong L^{\prime}$. Hence, $V^{\chi} \cdot S \subset \tilde{L}^{\prime}$ and $V^{\chi} \cdot \tilde{L} \subset \tilde{L}^{\prime}$.

Let $\chi^{-1}(g)=\chi\left(g^{-1}\right)$ for $g \in G$. Then, $\chi^{-1} \in \operatorname{Irr} G$ and $\chi^{-1} \cdot \chi=\chi \cdot \chi^{-1}=1_{G}$. Thus, $V^{\chi^{-1}} \cdot L^{\prime} \cong L$ and we have

$$
V^{\chi} \cdot \tilde{L} \supset V^{\chi} \cdot\left(V^{\chi^{-1}} \cdot \tilde{L}^{\prime}\right)=\left(V^{\chi} \cdot V^{\chi^{-1}}\right) \cdot \tilde{L}^{\prime}=\tilde{L}^{\prime}
$$

as desired.

(3) Let $L$ be an irreducible submodule of $M$. We shall define

$$
C_{L}=\left\{\chi \in \operatorname{Irr} G \mid L \cong V^{\chi} \cdot L\right\} .
$$

Since

$$
\left(V^{\chi \lambda}\right) \cdot L=V^{\chi} \cdot\left(V^{\lambda} \cdot L\right) \cong L, \text { for any } \chi, \lambda \in C_{L},
$$

we have $\chi \lambda \in C_{L}$ and $W(L)=\bigoplus_{\chi \in C_{L}} V^{\chi}$ is a subVOA of $V$.

Let

$$
H=H_{L}=\left\{g \in G \mid \chi(g)=1 \text { for all } \chi \in C_{L}\right\} \text {. }
$$

Then, $H$ is a subgroup of $G$ and $W(L)=\bigoplus_{\chi \in C_{L}} V^{\chi}=V^{H}$.

Note that the fact $W(L)=V^{H}$ also follows immediately from Quantum Galois theory (cf. [7]).

By the definition of $C_{L}$ and $\tilde{L}$, it is clear that

$$
u_{n} v \in \tilde{L} \text { for all } u \in W(L) \text { and } v \in \tilde{L} \text {. }
$$

Therefore, $\tilde{L}$ is a $V^{H}$-module. 
Next, we shall show that $H_{L}=H_{L^{\prime}}$ for any irreducible $V^{G}$-submodules $L$ and $L^{\prime}$ of $M$. By (1), there is a $\lambda \in \operatorname{Irr} G$ such that $L^{\prime} \cong \lambda L$ (or equivalently $\left.\tilde{L}^{\prime}=\lambda \tilde{L}=V^{\lambda} \cdot \tilde{L}\right)$.

Now let $\chi \in C_{L}$. Then, $\chi \tilde{L}^{\prime}=V^{\chi} \cdot \tilde{L}^{\prime}=V^{\chi} \cdot\left(V^{\lambda} \cdot \tilde{L}\right)=V^{\lambda} \cdot\left(V^{\chi} \cdot \tilde{L}\right)=$ $V^{\lambda} \cdot \tilde{L}=\tilde{L}^{\prime}$. Hence, $\chi \in C_{L^{\prime}}$ and we have $C_{L} \subset C_{L^{\prime}}$.

By symmetry, we must also have $C_{L} \supset C_{L^{\prime}}$. Therefore, $C_{L}=C_{L^{\prime}}$ and we have $H_{L^{\prime}}=H_{L}=H$. In particular, all $V^{G}$-homogeneous component are $V^{H}$-modules.

Now let us show that $\tilde{L}$ is an irreducible $V^{H}$-module.

Suppose not. Then, there exists an irreducible $V^{H}$-module $W$ such that $W \subsetneq \tilde{L}$. For any irreducible $V^{G}$-submodule $L$ of $W$, set

$$
N=V \cdot L=\sum_{\chi \in \operatorname{Irr} G} V^{\chi} \cdot L=\sum_{\chi \in C_{L}} V^{\chi} \cdot L+\sum_{\chi \notin C_{L}} V^{\chi} \cdot L .
$$

Note that $W=\sum_{\chi \in C_{L}} V^{\chi} \cdot L$ and $V^{\chi} \cdot L \not L$ for all $\chi \notin C_{L}$. Thus, $N \cap \tilde{L}=W$.

As $M$ is an irreducible $V$-module, $N=M$ and thus $N \cap \tilde{L}=\tilde{L}$. It is a contradiction and hence $\tilde{L}$ is an irreducible $V^{H}$-module.

Finally, we shall show that

$$
M=\bigoplus_{\lambda \in \operatorname{Irr} H}\left(V^{\lambda} \cdot L\right) .
$$

First, we shall note that

$$
H=\{g \in G \mid g v=v \text { for all } v \in W(L)\} \text { and } C_{L}=\left\{\chi \in \operatorname{Irr} G|\chi|_{H}=1_{H}\right\} .
$$

Let $\chi_{1}, \chi_{2} \in \operatorname{Irr} G$. Then,

$$
V^{\chi_{1}} \cdot \tilde{L}=\left.V^{\chi_{2}} \cdot \tilde{L} \Leftrightarrow V^{\chi_{1}} \cdot L \cong V^{\chi_{2}} \cdot L \Leftrightarrow \chi_{1}^{-1} \chi_{2} \in C_{L} \Leftrightarrow \chi_{1}\right|_{H}=\left.\chi_{2}\right|_{H} .
$$

Since $V^{\lambda}=\sum_{\chi \in \operatorname{Irr} G,\left.\chi\right|_{H}=\lambda} V^{\chi}$ for any $\lambda \in \operatorname{Irr} H$, we have

$$
V^{\lambda} \cdot \tilde{L}=\sum_{\chi \in \operatorname{Irr} G,\left.\chi\right|_{H}=\lambda} V^{\chi} \cdot \tilde{L}=V^{\chi} \cdot \tilde{L} .
$$

Hence, $M=\bigoplus_{\lambda \in \operatorname{Irr} H} V^{\lambda} \cdot \tilde{L}$.

(4) Suppose $\tilde{L}=L^{1} \oplus \cdots \oplus L^{m}$ where $L^{i} \cong L$. Then, $\chi \tilde{L}=\sum_{i} \chi L^{i}$. We shall show that the sum is in fact a direct sum.

Clearly, $\chi L^{i} \neq 0$ and $V^{\chi^{-1}} \cdot\left(V^{\chi} \cdot L^{i}\right)=\left(V^{\chi^{-1}} \cdot V^{\chi}\right) \cdot L^{i}=L^{i}$ for all $i$.

Thus, $\chi L^{i} \cap \chi L^{j}=0$ for all $i \neq j$ and we have $m(L)=m(\chi L)$ as required.

Next, we shall give a more precise structure of $M$.

Definition 3.8 ([1]). Let $G$ be a finite group and $\alpha$ a 2-cocycle. The twisted group algebra associated with $\alpha$ is the algebra 


$$
(\boldsymbol{C} G)_{\alpha}=\bigoplus_{x \in G} \boldsymbol{C} u_{x}
$$

with the multiplication defined by

$$
u_{x} u_{y}=\alpha(x, y) u_{x y} \quad \text { for } x, y \in G .
$$

Definition 3.9 ([1]). Let $G$ be a finite group. A projective representation of $G$, with respect to a 2-cocycle $\alpha$, is an algebra homomorphism $T:(\boldsymbol{C} G)_{\alpha} \rightarrow$ $G L(M)$ for some vector space $M$.

THeOREM 3.10. Let $M$ be an irreducible V-module and let $L$ be an irreducible $V^{G}$-submodule. Suppose that $M=\tilde{L}$, that is, all irreducible $V^{G}$-submodules of $M$ are isomorphic to $L$. Then, there is an irreducible projective representation $Q$ of $G^{*}=\operatorname{Irr} G$ such that

$$
M \cong Q \otimes_{C} L .
$$

Proof. The basic idea of the proof comes from a paper of Dong, Li and Mason [5]. We simply modify it to suit our case.

Let $L^{\chi}=V^{\chi} \cdot L \subset M$. Then, $L^{\chi} \cong V^{\chi} \hat{\otimes}_{V^{G}} L \cong L$ as $V^{G}$-modules and thus there is a $V^{G}$-isomorphism $f_{\chi}: L \rightarrow L^{\chi}$. Without loss, we shall choose $f_{1}=I d_{L}$.

Let $Y(\cdot, z): V \rightarrow$ End $M\left[\left[z, z^{-1}\right]\right]$ be the vertex operator for $M$. Then, $Y(\cdot, z) f_{\chi}$ defines an intertwining operator of type $\left(\begin{array}{c}L^{\lambda \chi} \\ V^{\lambda}\end{array}\right)$ since $V^{\lambda} \cdot\left(V^{\chi} \cdot L\right)$ $=\left(V^{\lambda} \cdot V^{\chi}\right) \cdot L=V^{\lambda \chi} \cdot L$ for any $\lambda, \chi \in \operatorname{Irr} G$.

As $L^{\lambda} \cong V^{\lambda} \hat{\otimes}_{V^{G}} L$, by the definition of tensor product, there is a $V^{G}$ homomorphism $f_{\chi, \lambda}: L^{\lambda} \rightarrow L^{\chi^{\lambda}}$ such that $Y(a, z) f_{\chi}(u)=f_{\chi, \lambda} Y(a, z) u$ for $a \in V^{\lambda}$ and $u \in L$. Note that if $L^{\lambda}=L^{\xi}$, we shall take $f_{\chi, \lambda}=f_{\chi, \xi}$.

Define $\hat{f}_{\chi}: M \rightarrow M$ by $\hat{f}_{\chi}(u)=f_{\chi, \lambda}(u)$ for $\lambda \in \operatorname{Irr} G$ and $u \in L^{\lambda}$. Then, $\hat{f}_{\chi}(u)$ is a well defined linear isomorphism of $M$ and $Y(a, z) \hat{f}_{\chi}(u)=\hat{f}_{\chi} Y(a, z) u$ for $u \in L$.

As $Y(\cdot, z)$ is the module vertex operator of $V$-module $M$, there is a positive integer $k>0$ such that the following associativities hold (cf. 2.4): For any $a, b \in V, \chi \in \operatorname{Irr} G$ and $u \in L$,

$$
\left(z_{0}+z_{2}\right)^{k} Y\left(a, z_{0}+z_{2}\right) Y\left(b, z_{2}\right) u=\left(z_{0}+z_{2}\right)^{k} Y\left(Y\left(a, z_{0}\right) b, z_{2}\right) u
$$

and

$$
\left(z_{0}+z_{2}\right)^{k} Y\left(a, z_{0}+z_{2}\right) Y\left(b, z_{2}\right) \hat{f}_{\chi} u=\left(z_{0}+z_{2}\right)^{k} Y\left(Y\left(a, z_{0}\right) b, z_{2}\right) \hat{f}_{\chi} u .
$$

Thus, we have 


$$
\begin{aligned}
\left(z_{0}+z_{2}\right)^{k} \hat{f}_{\chi} Y\left(a, z_{0}+z_{2}\right) Y\left(b, z_{2}\right) u & =\left(z_{0}+z_{2}\right)^{k} \hat{f}_{\chi} Y\left(Y\left(a, z_{0}\right) b, z_{2}\right) u \\
& =\left(z_{0}+z_{2}\right)^{k} Y\left(Y\left(a, z_{0}\right) b, z_{2}\right) \hat{f}_{\chi} u \\
& =\left(z_{0}+z_{2}\right)^{k} Y\left(a, z_{0}+z_{2}\right) Y\left(b, z_{2}\right) \hat{f}_{\chi} u \\
& =\left(z_{0}+z_{2}\right)^{k} Y\left(a, z_{0}+z_{2}\right) \hat{f}_{\chi}\left(Y\left(b, z_{2}\right) u\right)
\end{aligned}
$$

Multiply the equation by $\left(z_{0}+z_{2}\right)^{-k}$, we obtain

$$
\hat{f}_{\chi} Y\left(a, z_{0}+z_{2}\right) Y\left(b, z_{2}\right) u=Y\left(a, z_{0}+z_{2}\right) \hat{f}_{\chi}\left(Y\left(b, z_{2}\right) u\right)
$$

or

$$
\hat{f}_{\chi} Y\left(a, z_{1}\right) Y\left(b, z_{2}\right) u=Y\left(a, z_{1}\right) \hat{f}_{\chi}\left(Y\left(b, z_{2}\right) u\right)
$$

Since $M=V \cdot L$, we have

$$
\hat{f}_{\chi} Y\left(a, z_{1}\right) u=Y\left(a, z_{1}\right) \hat{f}_{\chi}(u) \text { for any } a \in V \text { and } u \in M
$$

Thus, $\hat{f}_{\chi}$ is a $V$-isomorphism.

Since $\left.\hat{f}_{\chi} \hat{f}_{\lambda}\right|_{L}$ and $\left.\hat{f}_{\chi \lambda}\right|_{L}$ are both $V^{G}$-homomorphism and $L$ is an irreducible $V^{G}$-module, $\left.\hat{f}_{\chi} \hat{f}_{\lambda}\right|_{L}$ is a scalar multiple of $\left.\hat{f}_{\chi \lambda}\right|_{L}$. In other words, we have a scalar $\alpha(\chi, \lambda) \in C^{*}$ such that $\hat{f}_{\chi} \hat{f}_{\lambda}(u)=\alpha(\chi, \lambda) \hat{f}_{\chi \lambda}(u)$ for any $u \in L$. On the other hand, for any $a \in V$ and $u \in L$, we have

$$
\hat{f}_{\chi} \hat{f}_{\lambda} Y(a, z) u=Y(a, z) \hat{f}_{\chi} \hat{f}_{\lambda}(u)=Y(a, z) \alpha(\chi, \lambda) \hat{f}_{\chi \lambda}(u)=\alpha(\chi, \lambda) \hat{f}_{\chi \lambda} Y(a, z)(u)
$$

Hence, $\hat{f}_{\chi} \hat{f}_{\lambda}=\alpha(\chi, \lambda) \hat{f}_{\chi \lambda}$ as an endomorphism of $M$.

Note that $\alpha(\chi, \lambda \xi) \alpha(\lambda, \xi)=\alpha(\chi, \lambda) \alpha(\chi \lambda, \xi)$ and $\alpha(1, \lambda)=\alpha(\lambda, 1)=1$. Thus, $\alpha(\chi, \lambda)$ is a 2-cocycle and $\left\{f_{\chi}\right\}$ defines an $C(\operatorname{Irr} G)_{\alpha}$-representation on $M$ where $C(\operatorname{Irr} G)_{\alpha}$ is the twisted group algebra associated with $\alpha$ (cf. Definition 3.8).

Since $f_{\chi}$ commutes with $Y(a, z)$ for all $a \in V^{G}, M$ is, in fact, a $C(\operatorname{Irr} G)_{\alpha} \otimes$ $V^{G}$-module. Thus,

$$
M=Q \otimes L
$$

where $Q$ is a $C(\operatorname{Irr} G)_{\alpha}$-module and $L$ is an irreducible $V^{G}$-module.

Since $\left.\hat{f}_{\chi}\right|_{L}=f_{\chi}: L \rightarrow L^{\chi}$ is a $V^{G}$-module isomorphism, there is an intertwining operator

$$
I^{\chi}(\cdot, z) \in I\left(\begin{array}{cc}
L & \\
V^{\chi} & L
\end{array}\right)
$$

such that

$$
Y(a, z)=f_{\chi} I^{\chi}(a, z) \quad \text { for } a \in V^{\chi} \text {. }
$$


Thus, the module vertex operator of $M$ can be rewritten as

$$
Y(a, z)=f_{\chi} \otimes I^{\chi}(a, z) \quad \text { for } a \in V^{\chi}
$$

where $f_{\chi}$ acts on $Q$ and $I^{\chi}(\cdot, z)$ acts on $L$.

Hence, $Q$ must be irreducible; otherwise there is a proper submodule $Q_{0} \subsetneq Q$. In this case, $Q_{0} \otimes L$ will be closed under the action of $Y(a, z)$. That means $Q_{0} \otimes L$ is a proper $V$-submodule of $M$. It is impossible as $M$ is irreducible.

THEOREM 3.11. Let $M$ be an irreducible $V$-module and $\left\{L_{1}, \ldots, L_{n}\right\}$ be the set of all inequivalence $V^{G}$-submodules of $M$. Then, there is a subgroup $H<G$ and irreducible $(G / H)^{*}=\operatorname{Irr}(G / H)$ projective representations $Q_{1}, \ldots, Q_{n}$ such that

$$
M=\bigoplus_{i} Q_{i} \otimes L_{i}
$$

Moreover, $\left\{Q_{i} \otimes L_{i}\right\}$ are irreducible $V^{H}$-modules.

Proof. By Part (3) of Theorem 3.7, there exist a subgroup $H<G$ such that $M=\bigoplus_{i=1}^{n} \tilde{L}_{i}$ where $\tilde{L}_{i}$ are $V^{G}$-homogeneous components of $M$ and $\tilde{L}_{i}$ are irreducible as $V^{H}$-modules.

Since $G$ is abelian, one can easily show

$$
V^{G}=\left(V^{H}\right)^{G / H} .
$$

Now, by Theorem 3.10, we know that

$$
\tilde{L}_{i}=Q_{i} \otimes L_{i}
$$

for some irreducible projective representations $Q_{i}$ of $(G / H)^{*}$. Hence,

$$
M=\bigoplus_{i} Q_{i} \otimes L_{i}
$$

as desired.

\section{Induced modules.}

In this section, we shall study the problem of extending an irreducible $V^{G}$-module $L$ to a $V$-module. In order to prevent some complicated technical details, we shall only consider the case that $L$ is contained in some $V$-module $M$. An induced module $X_{L}$ will be defined. We shall show that every irreducible $V$-module can be considered as a quotient module of some induced module.

As before, $L$ denotes an irreducible $V^{G}$-module. Suppose that there is a $V$-module $M$ such that $L \subset M$. 
Denote $L^{\chi}=V^{\chi} \hat{\otimes}_{V^{G}} L$. By our assumptions, $L^{\chi}$ is irreducible as a $V^{G}$-module; moreover, for any $\chi \in \operatorname{Irr} G$,

$$
V^{\chi} \cdot L \cong L^{\chi}
$$

where $V^{\chi} \cdot L=\operatorname{span}\left\{u_{n} v \mid u \in V^{\chi}, v \in L\right\} \subset M$.

Let $Y^{M}(\cdot, z)$ be the module vertex operator of $M$. Then, by restricting $Y^{M}$ to $V^{\chi}$, we obtain an intertwining operator

$$
I^{\chi, \lambda}(a, z)(u)=Y^{M}(a, z)(u) \in I\left(\begin{array}{c}
L^{\chi \lambda} \\
V^{\chi}
\end{array} L^{\lambda}\right) \quad \text { for } a \in V^{\chi}, u \in L^{\lambda}
$$

As a $V^{G}$-module, we define

$$
X_{L}=V \hat{\otimes}_{V^{G}} L=\bigoplus_{\chi \in \operatorname{Irr} G} V^{\chi} \hat{\otimes}_{V^{G}} L .
$$

and $\hat{Y}(\cdot, z): V \rightarrow$ End $X_{L}\left[\left[z, z^{-1}\right]\right]$ by

$$
\hat{Y}(u, z) v=I^{\chi, \lambda}(u, z) v \text { for } u \in V^{\chi}, v \in L^{\lambda} .
$$

Then, we have

THEOREM 4.1. $\left(X_{L}, \hat{Y}(\cdot, z)\right)$ is a V-module.

We shall denote the module $X_{L}$ by $\operatorname{Ind}_{V^{G}}^{V} L$ and call it an induced module of $L$ from $V^{G}$ to $V$.

REMARK 4.2. In [6], a general theory of induced modules for vertex operator algebras has been developed by Dong and Lin. Our definition of induced modules, on the other hand, follows the approach of Miyamoto in [13] which is slightly different from Dong and Lin's definition.

Lemma 4.3. The definition of $X_{L}$ is independent of the choice of the $V$-module $M$.

Proof. Let $\left(M^{1}, Y^{1}(\cdot, z)\right)$ and $\left(M^{2}, Y^{2}(\cdot, z)\right)$ be two $V$-modules such that both contain $L$ as an irreducible $V^{G}$-submodule. We shall show that $X_{L}^{1}$ defined by using $M^{1}$ and $X_{L}^{2}$ defined by using $M^{2}$ are isomorphic as $V$-modules.

Let $\hat{Y}^{1}(\cdot, z)$ and $\hat{Y}^{2}(\cdot, z)$ be the vertex operators of $X_{L}^{1}$ and $X_{L}^{2}$ respectively. Then, both $\left.\hat{Y}^{1}(\cdot, z)\right|_{V^{x}}$ and $\left.\hat{Y}^{2}(\cdot, z)\right|_{V x}$ are intertwining operators of type $\left(\begin{array}{cc}L^{\chi} \\ V^{\chi} & L\end{array}\right)$. As $L^{\chi}=V^{\chi} \hat{\otimes}_{V^{G}} L$, there exists a unique $V^{G}$-isomorphism $\psi_{\chi}$ such that

$$
\hat{Y}^{1}(u, z) v=\psi_{\chi} \hat{Y}^{2}(u, z) v
$$


By identifying the $L$ in $X_{L}^{1}$ and $X_{L}^{2}$, we may choose $\left\{\psi_{\chi}\right\}$ such that $\psi_{1}=i d_{L}$.

Now, define $\psi: X_{L}^{1} \rightarrow X_{L}^{2}$ by $\psi(v)=\psi_{\chi}(v)$ if $v \in L^{\chi}$. Clearly, $\psi$ is a linear isomorphism. We shall show that $\psi$ is also a $V$-isomorphism. By definition,

$$
\hat{Y}^{1}(u, z) \psi v=\psi \hat{Y}^{2}(u, z) v \text { for any } u \in V \text { and } v \in L .
$$

Since both $X_{L}^{1}$ and $X_{L}^{2}$ are $V$-modules, for any $a \in V^{\chi}, b \in V^{\lambda}$ and $v \in L$, there is a $k>0$ such that

$$
\left(z_{0}+z_{2}\right)^{k} \hat{Y}^{1}\left(a, z_{0}+z_{2}\right) \hat{Y}^{1}\left(b, z_{2}\right) \psi v=\left(z_{0}+z_{2}\right)^{k} \hat{Y}^{1}\left(Y\left(a, z_{0}\right) b, z_{2}\right) \psi v
$$

and

$$
\left(z_{0}+z_{2}\right)^{k} \hat{Y}^{2}\left(a, z_{0}+z_{2}\right) \hat{Y}^{2}\left(b, z_{2}\right) v=\left(z_{0}+z_{2}\right)^{k} \hat{Y}^{2}\left(Y\left(a, z_{0}\right) b, z_{2}\right) v .
$$

Now, by (4.8), (4.9) and (4.10), we have

$$
\left(z_{0}+z_{2}\right)^{k} \psi \hat{Y}^{2}\left(a, z_{0}+z_{2}\right) \hat{Y}^{2}\left(b, z_{2}\right) v=\left(z_{0}+z_{2}\right)^{k} \hat{Y}^{1}\left(a, z_{0}+z_{2}\right) \psi \hat{Y}^{2}(b, z) v
$$

and hence

$$
\psi \hat{Y}^{2}\left(a, z_{0}+z_{2}\right) \hat{Y}^{2}\left(b, z_{2}\right) v=\hat{Y}^{1}\left(a, z_{0}+z_{2}\right) \psi \hat{Y}^{2}(b, z) v
$$

or

$$
\psi \hat{Y}^{2}\left(a, z_{1}\right) \hat{Y}^{2}\left(b, z_{2}\right) v=\hat{Y}^{1}\left(a, z_{1}\right) \psi \hat{Y}^{2}\left(b, z_{2}\right) v
$$

Thus,

$$
\psi \hat{Y}^{2}\left(a, z_{1}\right) v=\hat{Y}^{1}\left(a, z_{1}\right) \psi v \text { for any } v \in V \hat{\otimes}_{V^{G}} L
$$

and $\psi$ is a $V$-isomorphism.

THEOREM 4.4. Let $M$ be an irreducible V-module. Then, $M$ is isomorphic to a quotient module of $\operatorname{Ind}_{V^{G}}^{V} L$ for some irreducible $V^{G}$-module $L$.

Proof. Let $L$ be an irreducible $V^{G}$-submodule of $M$. Then, by Theorem 3.7,

$$
M=\sum_{\chi \in \operatorname{Irr} G} V^{\chi} \cdot L=\sum_{\chi \in \operatorname{Irr} G} L^{\chi}
$$

Since $V^{\chi_{i}} \hat{\otimes}_{V^{G}} L \cong L^{\chi}$, there is a $V^{G}$-homomorphism

$$
\psi_{\chi}: V^{\chi_{i}} \hat{\otimes}_{V^{G}} L \rightarrow L^{\chi} .
$$

Define $\psi: \operatorname{Ind}_{V^{G}}^{V} L \rightarrow M$ such that $\left.\psi\right|_{V^{\chi} \hat{\otimes}_{V G} L}=\psi_{\chi}$. By the same argument as in Lemma 4.3, one can show that $\psi$ is a $V$-homomorphism. As $M$ is an irreducible $V$-module, $\psi$ must be surjective and hence $M$ is isomorphic to a quotient module of $\operatorname{Ind}_{V^{G}}^{V} L$. 
The next theorem is a generalization of the rationality of framed (and code) VOA (cf. [2] and [13]).

THEOREM 4.5. Let $V$ be a simple VOA and $G<$ Aut $V$ a finite abelian group. Suppose that $V^{G}$ is rational and $\sum_{W} N_{V \times L}^{W}=1$ for any irreducible $V^{G}$-modules $L$. Then, $V$ is also rational.

Proof. We shall show that every $V$-module is completely reducible. Let $M$ be a $V$-module and choose a $V$-submodule $W \subset M$ such that $W$ is maximal among all submodules of $M$ which are direct sums of irreducible $V$-submodules. We shall show that $W=M$.

Suppose $W \neq M$. Then, there is an irreducible $V^{G}$-submodule $L$ of $M$ such that $W \cap L=0$ as $V^{G}$ is rational. Let $\tilde{L}$ be the sum of all irreducible submodules of $M$ isomorphic to $L$ and $\tilde{U}=V \cdot L$. By the proof of Theorem 3.11, there is an $H<G$ such that

$$
U^{\prime}=\tilde{U} \cap \tilde{L} \cong Q \otimes L \text { as } V^{H} \text {-modules }
$$

where $Q$ is a projective representation of $\operatorname{Irr}(G / H)$ and $V^{H}=\bigoplus_{V^{\chi} \cdot L \cong L} V^{\chi}$, $\chi \in \operatorname{Irr}(G)$.

Since $L \nsubseteq W$, by Theorem 3.11, there is a proper submodule $Q_{0}$ of $Q$ such that

$$
W \cap U^{\prime} \cong Q_{0} \otimes L \quad \text { as } V^{H} \text {-modules. }
$$

Since $\boldsymbol{C}$ is an algebraically closed field, we have $Q=Q_{0} \oplus Q_{1}$ for some $Q_{1}$. By the proof of Theorem 3.11, we know that $Q_{1} \otimes L \subset \tilde{U}$ is a $V^{H}$-module. Now, let

$$
X=\sum_{\chi \in \operatorname{Irr} G} V^{\chi} \cdot\left(Q_{1} \otimes L\right) .
$$

Then, $X$ is a $V$-module and by the definition of $V^{H}$, we have $X \cap \tilde{L}=Q_{1} \otimes L$. Thus, $X \cap W \cap \tilde{L}=0$.

Since all $V^{G}$-homogeneous component of $X$ are permuted transitivity by $V^{\chi}$ (cf. Theorem 3.7), we have $X \cap W=0$.

Now let $Y$ be an irreducible $V$-submodule of $X$. Then,

$$
M \supset Y \oplus W \supsetneq W .
$$

It contradicts the maximality of $W$. Hence, $W=M$.

Now, by Theorem 4.4, it is easy to see that $V$ has only finitely many inequivalent irreducible modules. 
REMARK 4.6. Since $\operatorname{Ind}_{V^{G}}^{V} L$ is completely reducible, every quotient module is isomorphic to some submodule. Hence, every irreducible $V$-module is isomorphic to some submodule of an induced module.

\section{References}

[1] C. W. Curtis and I. Reiner, Method of Representation Theory, Vol. 1, John Wiley \& Son, 1981.

[2] C. Dong, R. L. Griess Jr. and G. Höhn, Framed, vertex operator algebras, codes and the moonshine module, Comm. in Math. Phys., 193 No. 2 (1998), pp. 407-448.

[3] C. Dong and J. Lepowsky, Generalized vertex algebras and relative vertex operators, Progress in Math. Vol. 112, Birkhäuser, Boston 1993.

[4] C. Dong, H. Li and G. Mason, Regularity of rational vertex operator algebras, Adv. Math., 132 (1997), 148-166.

[ 5 ] C. Dong, H. Li and G. Mason, Simple, currents and extensions of vertex operator algebras, Comm. Math. Phys., 180 (1996), 671-707.

[ 6 ] C. Dong and Z. Lin, Induced modules for vertex operator algebras, Comm. Math. Phys., 179 (1996), 154-184.

[ 7 ] C. Dong and G. Mason, On Quantum Galois Theory, Duke Math. J., 86 (1997), no. 2, $305-$ 321.

[8] I. B. Frenkel, Y. Huang and J. Lepowsky, On axiomatic approaches to vertex operator algebras and modules, Mem. Amer. Math. Soc., 104, 1993.

[9] I. B. Frenkel, J. Lepowsky, and A. Meurman, Vertex Operator Algebras and the Monster, Pure and Applied Math., Vol. 134, Academic Press, 1988.

[10] H. Li, Local systems of vertex operators, vertex superalgebras and modules, J. Pure Appl. Algebra, 109 (1996), no. 2, 143-195.

[11] H. Li, Representation theory and tensor product theory of vertex operator algebras, Ph.D. dissertation, Rutgers University, 1994.

[12] M. Miyamoto, Binary codes and vertex operator (super)algebras, J. Algebra, 181 (1996), 207-222.

[13] M. Miyamoto, Representation theory of code VOA and construction of VOA, preprint.

[14] Y. Zhu, Vertex operator algebras, elliptic functions and modular form, Ph.D. dissertation, Yale University, 1990.

\section{Ching Hung LAM}

Department of Mathematics

National Cheng Kung University

Tainan, Taiwan 701, R.O.C. 